livraisons

d'Histoire

de l'Architecture

\section{Livraisons de l'histoire de l'architecture}

$27 \mid 2014$

Varia II

\title{
De Francesco Borromini à Paolo Portoghesi. L'église de la Sacra Famiglia à Salerne
}

From Francesco Borromini to Paolo Portoghesi : the Sacra Familia church at Salerne

Von Francesco Borromini bis Paolo Portoghesi: die Kirche der Sacra Familia in Salerno

\section{Benjamin Chavardes}

\section{OpenEdition}

\section{Journals}

Édition électronique

URL : http://journals.openedition.org/lha/352

DOI : 10.4000/lha.352

ISSN : 1960-5994

Éditeur

Association Livraisons d'histoire de l'architecture - LHA

Édition imprimée

Date de publication : 10 juin 2014

Pagination : 21-31

ISSN : 1627-4970

Référence électronique

Benjamin Chavardes, « De Francesco Borromini à Paolo Portoghesi. L'église de la Sacra Famiglia à Salerne », Livraisons de l'histoire de l'architecture [En ligne], 27 | 2014, mis en ligne le 10 juin 2016, consulté le 19 avril 2019. URL : http://journals.openedition.org//ha/352 ; DOI : 10.4000//ha.352 
Par Benjamin Chavardes

\section{DE FRANCESCO BORROMINI À PAOLO PORTOGHESI. L'ÉGLISE DE LA SACRA FAMIGLIA À SALERNE}

En 1968, le père Nicola Roberto cède à la Societa Autostrade une parcelle de terrain pour la construction d'une portion d'autoroute. En échange, il demande un financement pour la construction d'une nouvelle église. La conception de l'église de la Sacra Famiglia est confiée à l'architecte Paolo Portoghesi et son associé, l'ingénieur Vittorio Gigliotti ${ }^{1}$. Elle sera consacrée en 1974. Après le Concile Vatican II (1962-1965), ce projet a pour principale ambition de réaliser la nouvelle liturgie et ainsi de favoriser "la participation active des fidèles" comme le prescrit le Sancrosanctum Concilium ${ }^{2}$. Le projet doit donc s'inscrire dans la continuité de l'Église catholique romaine tout en proposant une nouvelle solution architecturale.

\section{Rupture et continuité}

Au même moment, la disparition des maîtres de l'architecture moderne, comme Le Corbusier en 1965 ainsi que Walter Gropius et Mies van der Rohe en 1969, laisse orphelins les architectes qu'ils ont formés. Cette "génération de l'incertitude " ${ }^{3}$, âgée de 40 à 60 ans, entre dans une période de doute, qui est accentuée par la crise économique de 1973. Elle est partagée entre l'héritage des maîtres et le nécessaire renouvellement de la pensée architecturale. En Italie, de nombreuses œuvres d'art sont endommagées par les inondations record de Florence en $1966^{4}$. Les images du désastre marquent profondément les esprits et sensibilisent particulièrement le public à la nécessite de protéger cet héritage historique. Le lien au passé dans le processus de projet devient une question idéologique majeure $\mathrm{du}$ débat architectural qui trouvera une expression particulière avec l'inauguration de la Strada Novissima à l'occasion de la première biennale d'architecture de Venise, en 1980. Cette exposition, dirigée par Paolo Portoghesi, a pour titre : la presenza

1. Vittorio Gigliotti est né à Salerne en 1921. Après avoir obtenu un diplôme d'ingénieur civil à Naples en 1947, il s'associe à Bruno Zevi, avant de fonder en 1964 le Studio di Porta Pinciana à Rome avec Paolo Portoghesi.

2. Constitution de la liturgie sacrée Sacrosanctum Concilium, promulguée par le pape Paul VI le 4 décembre 1963. Chapitre VII, L'art sacré et le matériel de culte, p. 124.

3. Francesco Dal Co et Mario Manieri-Elia, "La génération de l'incertitude", dans L'Architecture d'aujourd'hui, $\mathrm{n}^{\circ} 181,1975$, p. 34-56.

4. Au début du mois de novembre 1966, les eaux de l'Arno envahissent les rues de Florence. Les peintures conservées dans les sous-sols des Offices sont endommagées comme les collections de la Bibliothèque nationale et un grand nombre d'œuvres d'art. 
del passato $^{5}$. Dans ce contexte, la question posée à Portoghesi pour le projet de Salerne est de savoir comment allier continuité et rupture ou plutôt comment renouer le lien avec le passé en proposant une nouvelle architecture.

\section{Borromini comme maître}

Paolo Portoghesi est né à Rome en 1931. Il grandit dans un immeuble de la via Monterone dans le cœur historique de Rome, entre le Panthéon et l'église Sant'Ivo alla Sapienza conçu par Francesco Borromini. La lanterne de cette église constitue un élément clé du paysage quotidien du jeune Portoghesi. Ce dernier déclare :

«Plutôt que de le choisir, je pense que Borromini a été un maitre qui m’a été assigné par le destin. Depuis l'enfance, il m'a attiré de façon intense et passionnante ${ }^{6}$."

À 14 ans, il écrit un petit essai sur Borromini qui est le premier d'une importante série ${ }^{7}$. Ainsi, en 1953, le jeune étudiant en architecture de 22 ans publie un premier article sur Borromini dans la revue Civiltà delle Macchine ${ }^{8}$. Entre 1953 et 1967, date du tricentenaire de la mort de Borromini, Portoghesi publie vingt et un articles sur Borromini et deux monographies ${ }^{9}$, ainsi qu'une édition de l'Opus architectonicum $^{10}$. Il est également utile d'ajouter à cette liste ses publications sur l'architecture baroque, en particulier Roma barocca ${ }^{11}$ publié en 1966, qui font une place généreuse au maître baroque tessinois.

Le portrait de Borromini dans l'Opus architectonicum (ill. 1) est aujourd'hui accroché dans l'entrée de l'agence de Portoghesi à Calcata, au nord de Rome. Ce portrait siège également sur son bureau aux cotés de celui de son père comme deux figures tutélaires ${ }^{12}$. Et lorsqu'en 1983, Francesco Moschini, le directeur de la galleria d'Arte e Architettura Moderna à Rome, demande à Portoghesi de réaliser un autoportrait, c'est le même portrait qui lui sert de modèle. Il reprend la forme du cadre, la posture et la composition dans une imitation assumée et revendiquée par

5. La Biennale, Prima Mostra Internazionale di Architettura, La Presenza del passato, 1980, Venise, La Biennale di Venezia/Electa.

6. Mario Pisani, Dialogo con Paolo Portoghesi per comprendere l'architettura, Rome, Officina, 1969, p. 18, traduction de l'auteur.

7. Diego Lama, "Paolo Portoghesi ", Cemento Romano, Naples, CLEAN, 2010, p. 158.

8. Paolo Portoghesi, "Borromini in ferro", Civilta delle Macchine, n 2, 1953, p. 50-53.

9. Paolo Portoghesi, Borromini nella cultura europea, Rome, Officina, 1964. Paolo Portoghesi, Borromini. Architettura come linguaggio, Milan, Electa, 1967.

10. Paolo Portoghesi, Edizioni dell'Opus Architectonicum di Francesco Borromini, Rome, Edizioni dell'Elefante, 1964.

11. Paolo Portoghesi, Roma barocca, Storia di una civilta architettonica, Rome, Carlo Bestetti Edizioni d'Arte, 1966.

12. Ce fait a été relevé par l'auteur à l'occasion de visites au studio de Paolo Portoghesi à Calcata en mars 2010 et juillet 2011 . 


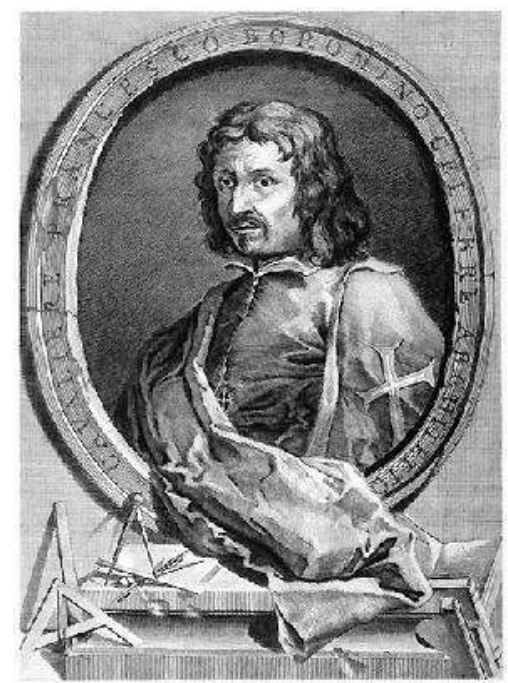

Ill. 1 : Anonyme, "Portrait de Francesco Borromini ", dans Francesco Borromini, Opus architectonicum, Rome, 1725 .

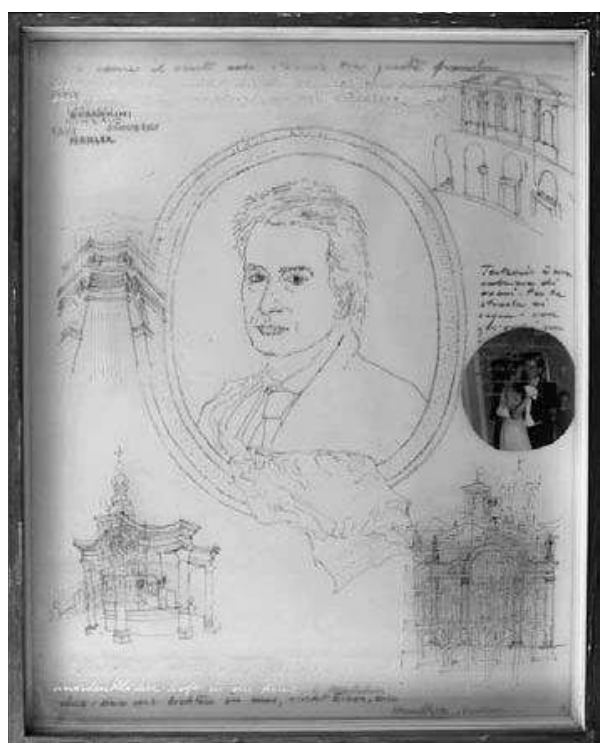

Ill. 2 : Paolo Portoghesi, Autoritratto per Francesco Moschini, Rome, 1983. Techniques mixtes : graffiti et collage sur verre (3 en série) et carton, $50 \times 40 \times 6 \mathrm{~cm}$. [Collection permanente Francesco Moschini e Gabriel Vaduva, galleria A.A.M. Architettura Arte Moderna]. En ligne : http://www.aamgalleria.it/ cfm-collezione.php?id=2130-Paolo-Portoghesi\&img=11 [consulté le 24.12.2013].

la présence de la lanterne de Sant'Ivo alla Sapienza dans l'angle inférieur gauche (ill. 2). 
Paolo Portoghesi débute son activité d'architecte en 1957. Il développe parallèlement son activité d'historien et d'architecte.

\section{Construction géométrique}

Le dessin d'étude de l'église de Salerne conservé par le centre Georges Pompidou (ill. 3) permet de comprendre les principes ayant présidé la construction géométrique. Il est nécessaire de distinguer tout d'abord deux petits croquis qui reprennent les plans de Sant'Ivo alla Sapienza et de San Carlino allo Quattro Fontane, deux œuvres majeures de Borromini. Comme il l'indique avec une flèche, il déduit du plan de La Sapienza l'utilisation de six centres alliés en deux systèmes de trois (6, numéros de la création et 3, pour la Sainte-Famille). Ces six centres sont disposés en spirale de façon à transposer en plan l'hélix de la lanterne de $\mathrm{La}$ Sapienza. La spirale exprime ainsi dans le même temps l'évolution d'une force et la volonté d'ouverture, une expansion vers l'extérieur symbolisant la nouvelle liturgie, comme indiqué par les flèches sur le croquis.

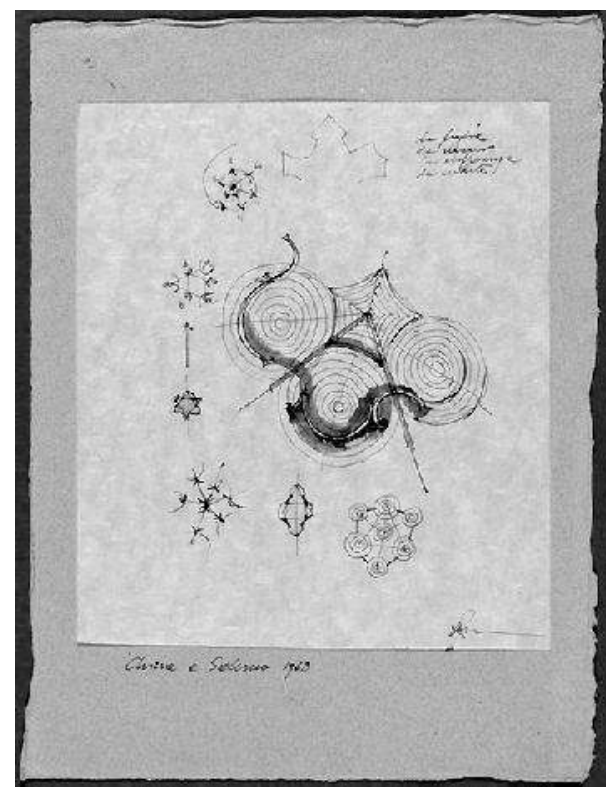

Ill. 3 : Paolo Portoghesi, Église de la Sacra Famiglia, Salerne. Étude formes géométriques, 1968, encre sépia sur papier [Centre Georges Pompidou. Inv. : AM 2009-2-876].

Portoghesi explique dans son "autobiographie scientifique" Le inibizioni dell'architettura moderna, que c'est par l'étude de l'œuvre de Borromini qu'il a compris que le cylindre à base elliptique, considéré comme l'enveloppe du corps humain, peut synthétiser la présence du corps ${ }^{13}$.

13. Paolo Portoghesi, Le inibizioni dell'architettura moderna, Bari, Laterza, 1974, p. 84-85.

Livraisons d'bistoire de l'architecture $n^{\circ} 27$ 


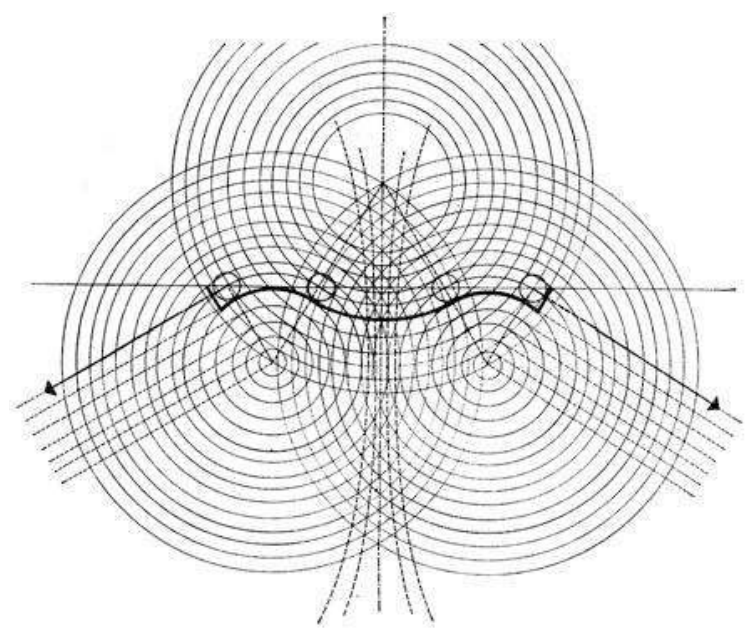

Ill. 4 : Paolo Portoghesi, «Interprétation spatiale de la façade de San Carlo alle Quattro Fontane de Francesco Borromini ", dans Paolo Portoghesi, Borromini. Architettura come linguaggio, Electa, Milano, 1967 , p. 300.

Ainsi il utilise des plans analytiques pour étudier les travaux de Borromini en dessinant des séries de cercles concentriques ${ }^{14}$ (ill. 4). Pour Portoghesi, la force qu'exerce un objet architectural sur un individu passe en particulier par sa matérialité. En ce sens, le lien entre structure architecturale et espace est comparable à celui qui existe entre un pôle magnétique et le champ qu'il génère. Cette notion de champ permet ainsi à Portoghesi de ne plus considérer l'espace comme une simple contre-forme de l'enveloppe architecturale. Il appelle cette conception la théorie de l'espace comme système de lieux. Suivant cette méthode, dans l'église de Salerne, six centres génèrent six champs réalisés sous forme de séries de cercles concentriques qui dessinent la composition spatiale de l'édifice (ill. 5).

L'église de la Sacra Famiglia marque l'aboutissement de ce travail de recherche débuté dix ans plus tôt, en 1959, avec la casa Baldi en périphérie de Rome. L'amitié entre Portoghesi et Christian Norberg-Schulz n'est pas étrangère à ce travail. Le travail sur l'architecture baroque et le Genius Loci nourrit la réflexion de Portoghesi ${ }^{15}$.

\section{Figures du langage architectural}

Dans sa monographie de $1967^{16}$, Portoghesi identifie dix figures dans le langage architectural de Borromini : la métaphore, l'antithèse, l'oxymore, l'ironie, l'allégorie, la personnification, la gradation, l'anastrophe, l'épiphore et l'ellipse.

14. Paolo Portoghesi, Borromini. Architecture, langage, Paris, Vincent, Fréal \& C Ce, 1969, p. 48, 66, 169, 172, 297, 300, 387

15. Christian Norberg-Schulz, Genius Loci, Milan, Electa, 1979, 214 p

16. Paolo Portoghesi, op. cit., 1967, p. 376-378. 


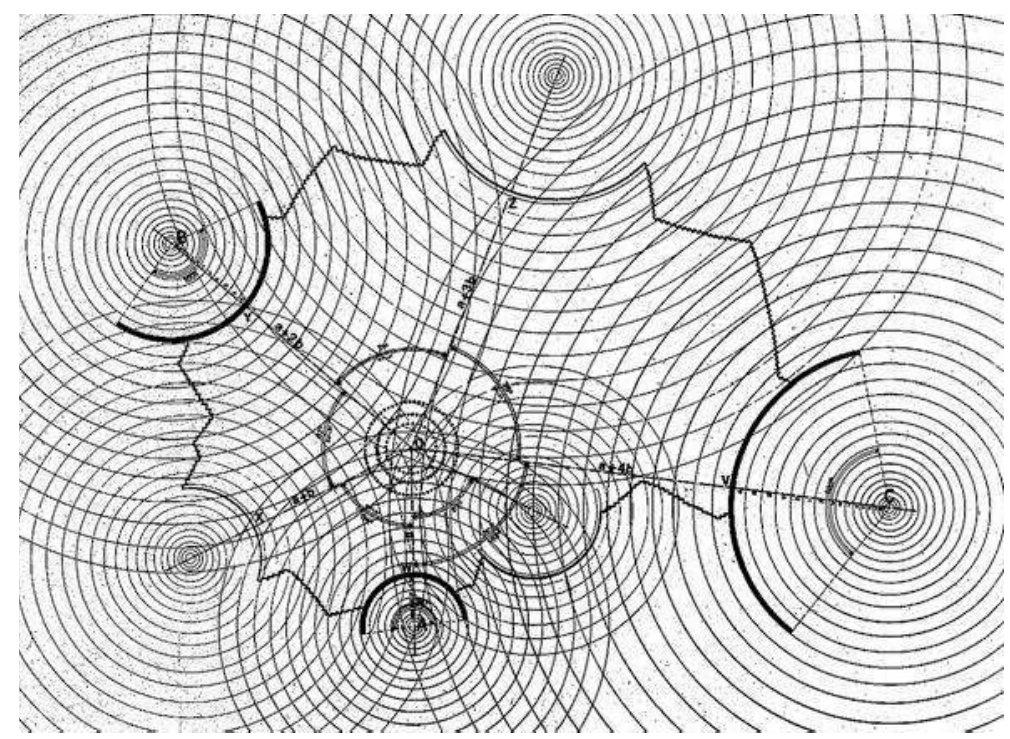

Ill. 5 : Paolo Portoghesi, "Géométrie de l'église de la Sacra Famiglia ", dans Maria Ercadi, Petra Bernitsa, Paolo Portoghesi, Skira, Milano, 2006, p. 60.

Dans son église, il en utilise au moins deux. La première est la métaphore naturelle. Borromini utilisait des sculptures ou des éléments d'architecture inspirés par les formes de la nature. La lanterne de La Sapienza par exemple imite la forme de certains coquillages. Nous savons en consultant l'inventaire réalisé à sa mort qu'il y avait plusieurs types de coquillages présent dans l'atelier du maitre ${ }^{17}$. L'hélix de la lanterne fait également référence à la tour de Babel et à l'idée de croissance.

À Salerne, Portoghesi utilise la métaphore de l'arbre. L'église est comparée à un arbre. Les cercles concentriques illustrent la coupe d'un arbre. Ainsi l'église contient trois arbres à l'intérieur afin de former la voûte: "all'ombra di tre grande alberi", à l'ombre de trois grands arbres, comme il l'écrit et le dessine dans un dessin d'étude (ill. 6) et "il sole », le soleil, pour parler de l'éclairage zénithal.

L'autre figure du langage est l'antithèse c'est-à-dire la combinaison d'éléments opposés. L'exemple le plus évident chez Borromini est l'opposition concave convexe que l'on trouve parmi d'autre dans la façade de San Carlino alle Quattro Fontane. Portoghesi utilise cette figure et utilise également une opposition entre les murs rectilignes et les murs courbes et entre surface et gradins (ill. 7). La toiture en terrasses trouve son origine première dans La Sapienza de Borromini. La référence au tombeau de la famille Brion ${ }^{18}$ de Carlo Scarpa semble évidente mais celui-ci est conçu au même moment que l'église de Salerne, entre 1969 et 1978. Portoghesi

17. Paolo Portoghesi, op. cit., 1964, p. 397.

18. Tombeau de la famille Brion, Carlo Scarpa, 1969-1978, San Vito d'Altivole (Trevise, Italie), en collaboration avec G. Pietropoli et C. Maschietto. 


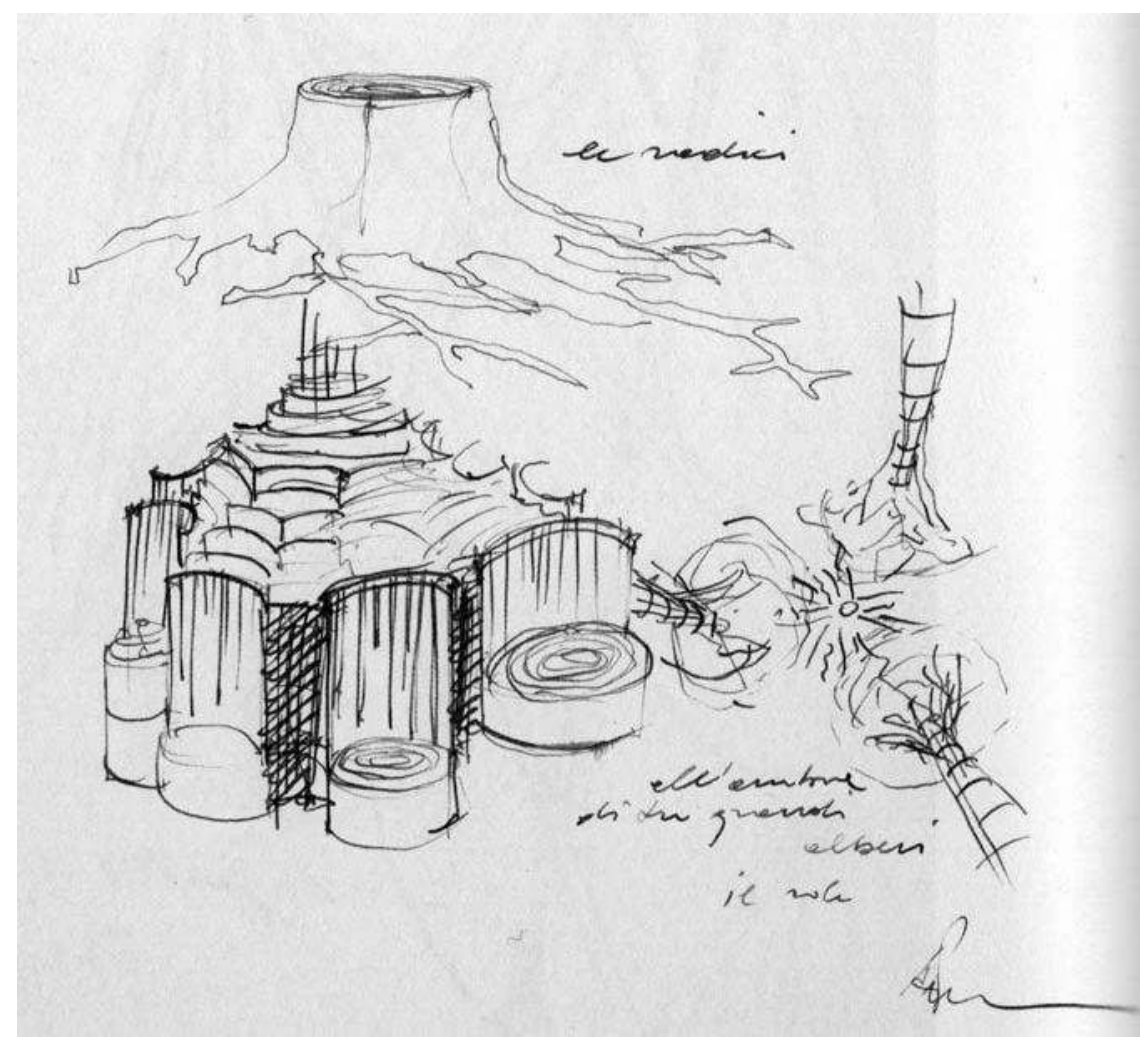

Ill. 6: Paolo Portoghesi, "Étude pour l'église de la Sacra Famiglia», dans Maria Ercadi, Fabrizio Da Col, Paolo Portoghesi disegni 1949-2003, Motta, Milano, 2003, p.150.

utilise ce toit pour la première fois dans la casa Bevilacqua ${ }^{19}$ à Gaeta et on le trouve ensuite dans la grande mosquée de Rome ${ }^{20}$. De plus, Portoghesi fait également référence à l'image en gradin d'un ancien théâtre qui constitue un autre modèle d'assemblée. Les gradins se développent jusqu'au sommet, pour une réinterprétation de la lanterne de Borromini (ill. 8).

\section{La lumière}

Portoghesi analyse le travail sur la modénature des corniches dans l'œuvre de Borromini. Il y voit la volonté de travailler la lumière de façon à générer des pulsations lumineuses, avec des successions d'ombres et de lumières. Pour nommer ce

19. Casa Bevilacqua, conçue par Paolo Portoghesi et Vittorio Gigliotti, 1964-1973, Gaeta (Latina, Italie).

20. Mosquée et centre culturel islamique de Rome, conçue par Paolo Portoghesi, Vittorio Gigliotti et Sami Mousawi, 1974-1995. 


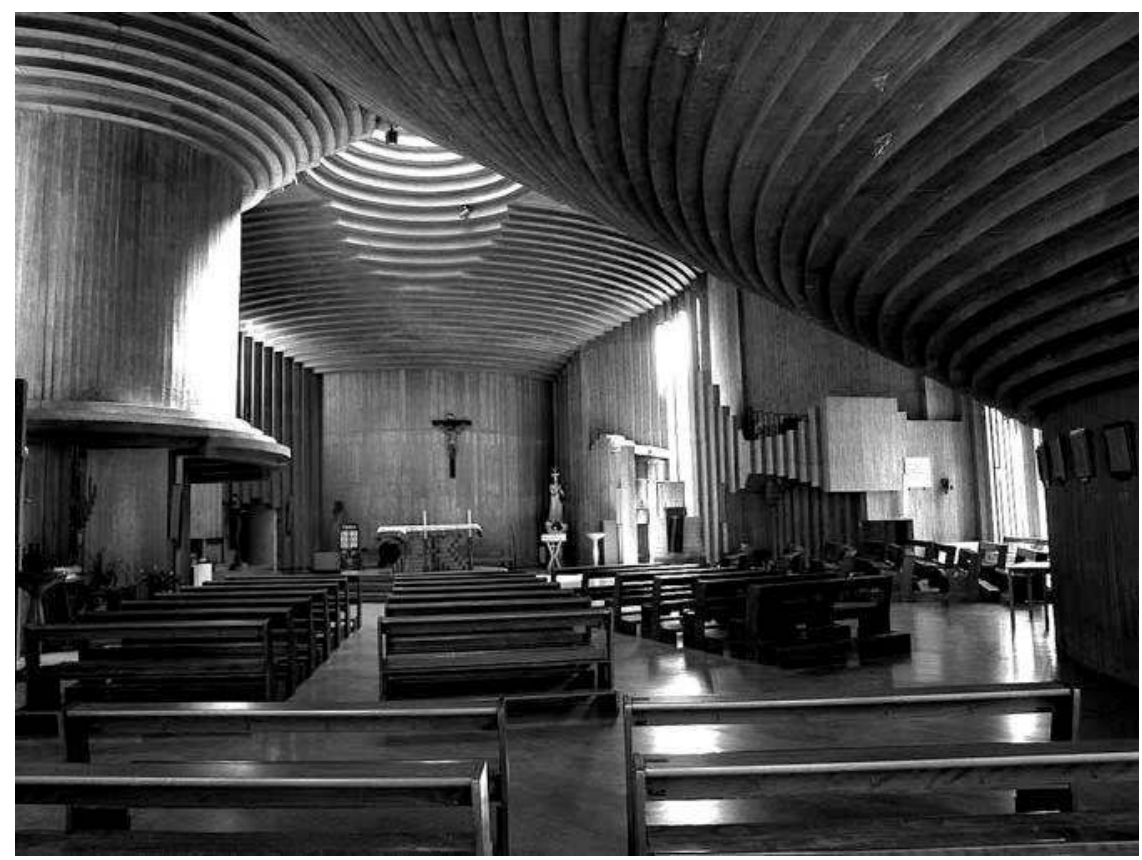

Ill. 7 : Paolo Portoghesi \& Vittorio Gigliotti, Vue intérieure de l'église de la Sacra Famiglia, Salerne. Cl. Benjamin Chavardés, 2011.

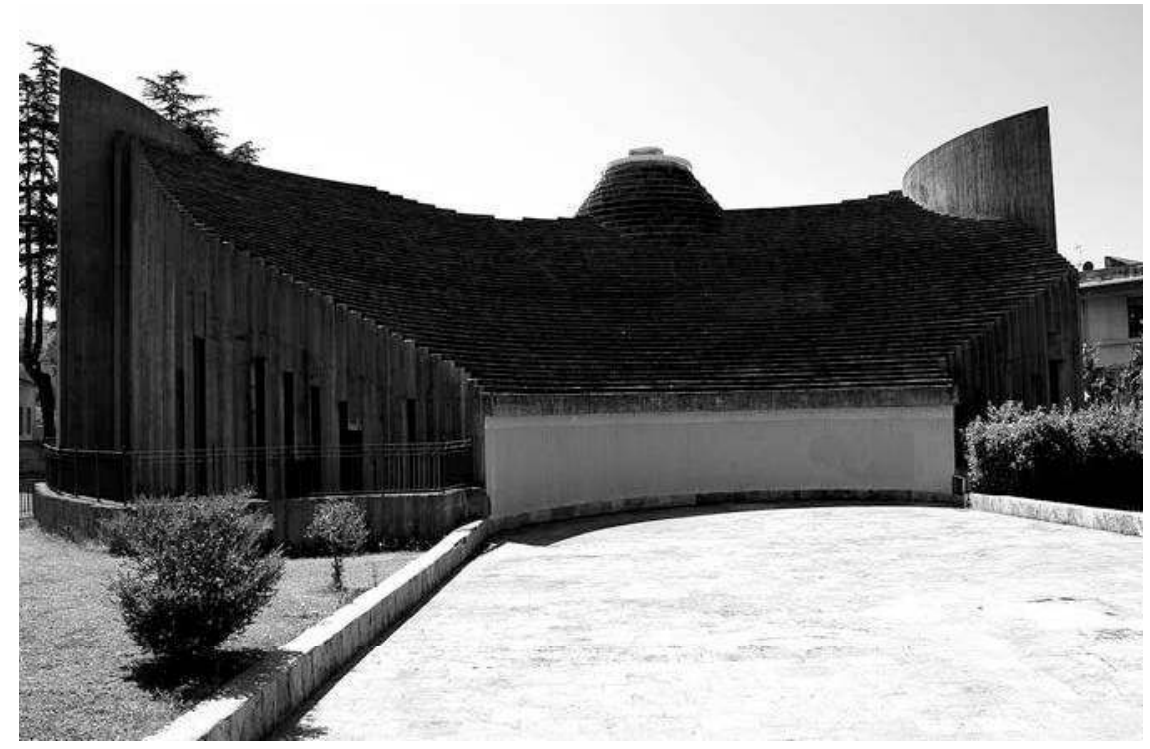

Ill. 8 : Paolo Portoghesi \& Vittorio Gigliotti, Vue extérieure de l'église de la Sacra Famiglia, Salerne. Cl. Benjamin Chavardés, 2011. 
processus, il utilise un terme généralement utilisé pour le travail de Léonard de Vinci. Il parle de sfumato. Dans l'église de la Sacra Famiglia, le processus est amplifié par l'utilisation d'une voûte en gradins. Celle-ci permet également de réaliser la troisième dimension du travail de l'espace comme système de lieux. Ainsi Portoghesi génère un rythme lumineux graduel et dégradé qui diffuse la lumière depuis l'unique éclairage zénithal qui surplombe l'autel. Celui-ci est ainsi mis en lumière au centre de l'édifice. L'éclairage zénithal est complèté par des fenêtres périphériques qui respectent le module de 25 centimètres de l'édifice. Elles permettent d'accroître l'effet d'aspiration vers l'extérieur.

\section{La continuité plastique et spatiale}

Chez Borromini, la continuité plastique et spatiale constitue le vecteur de l'unité. Ainsi il est possible, d'un seul coup d'œil, d'embrasser l'ensemble de l'espace interne. C'est également pour cela qu'il abandonne les éléments verticaux dans les structures horizontales. Chez Portoghesi, la continuité se manifeste dans la connexion entre les éléments verticaux et horizontaux (ill. 9 et 10). Cela constitue le résultat de ses recherches sur la théorie de l'espace comme système de lieux. Depuis le plan jusqu'à la toiture, toute la composition est le résultat d'un processus d'extrusion.

La continuité entre intérieur et extérieur est traitée par la construction géométrique mais également dans la contraction et la dilatation de l'espace qui met en scène le rôle central de l'autel dans la liturgie. Dans sa monographie de 1967, Portoghesi précise que :

«Bien que souvent Borromini aborde le problème de la configuration interne du bâtiment indépendamment de celle de son aspect extérieur, il a également formulé de façon claire l'hypothèse d'une unité organique entre l'espace intérieur et l'espace extérieur ${ }^{21}$."

Contrairement à Borromini, dans l'église de Portoghesi la conception des façades n'est pas distincte de l'espace intérieur. Aucun espace intermédiaire ne vient s'insérer et la façade épaisse disparaît, ce qui permet de conserver une continuité entre intérieur et extérieur. D'un autre côté, nous trouvons un traitement de l'angle, que Borromini utilise dans la maison des oratoriens de Rome, avec un angle convexe qui construit l'espace extérieur. Si Portoghesi utilise le travail de Borromini pour traiter la dualité continuité discontinuité, c'est parce que le lien avec le passé est déjà présent dans l'œuvre de l'architecte baroque. Ce dernier pose la même question à propos de l'héritage de Michel-Ange. Ce que Portoghesi recherche et utilise de Borromini et de l'architecture baroque, c'est le mouvement.

21. Paolo Portoghesi, op. cit., 1969, p. 389. 


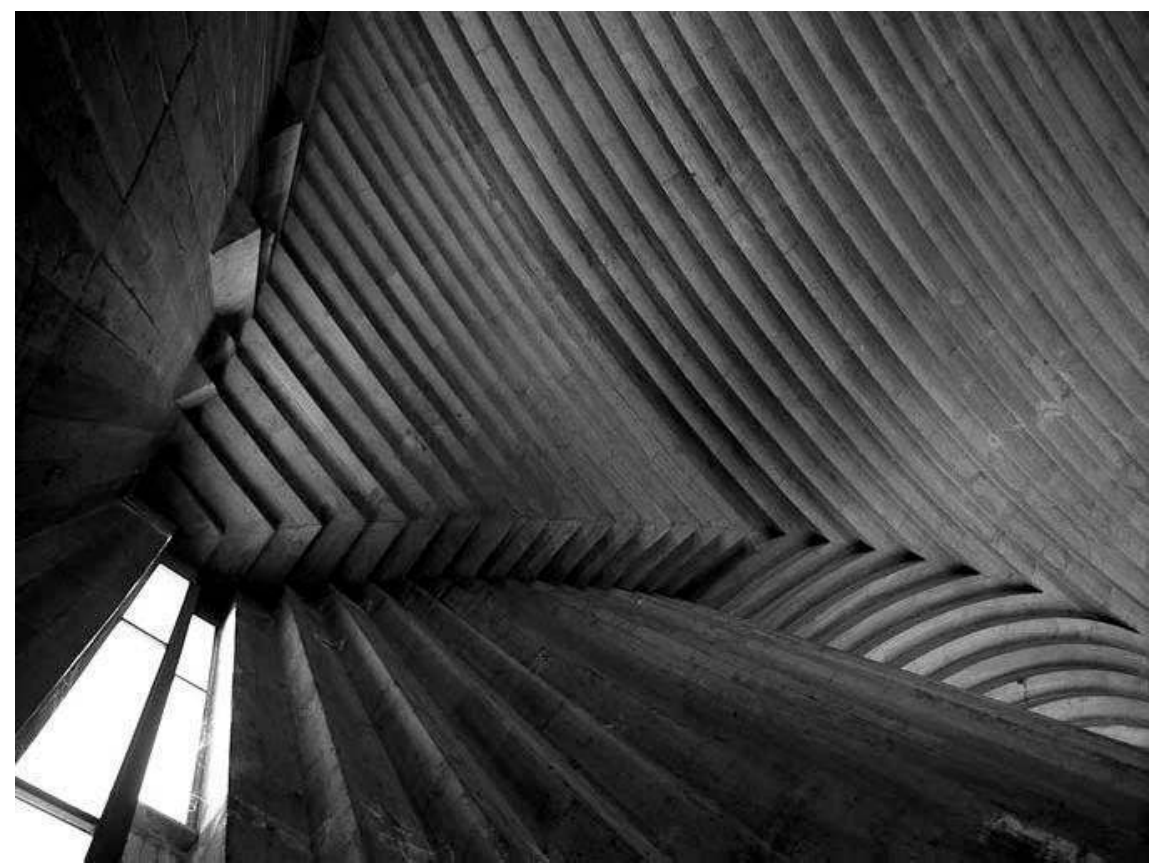

Ill. 9: Paolo Portoghesi \& Vittorio Gigliotti, Détail du plafond de l'église de la Sacra Famiglia, Salerne. Cl. Benjamin Chavardés, 2011.

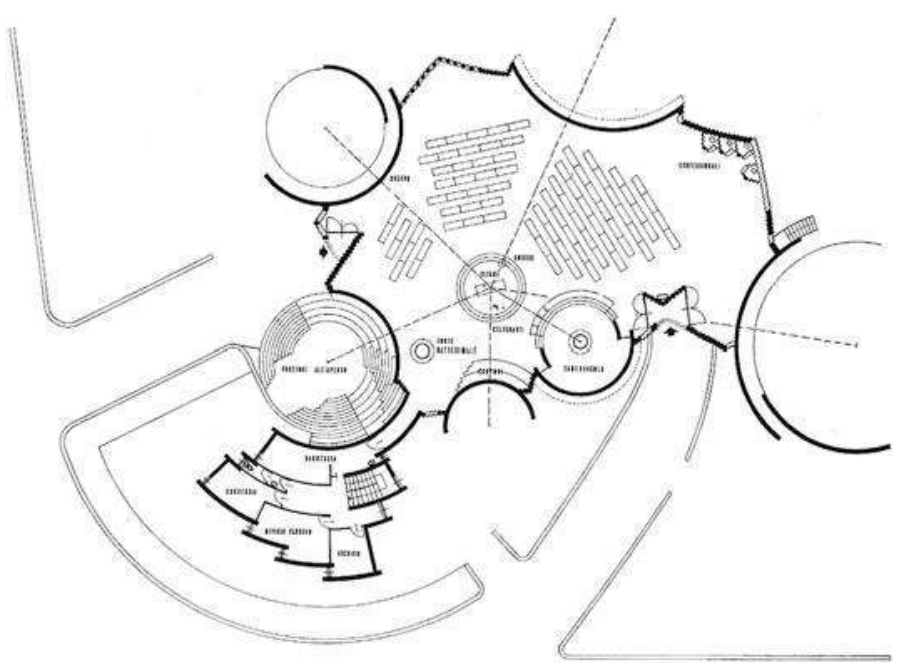

Ill. 10 : Paolo Portoghesi \& Vittorio Gigliotti, «Plan de l'église de la Sacra Famiglia », dans Maria Ercadi, Petra Bernitsa, Paolo Portoghesi, Skira, Milano, 2006, p. 61. 
Il a la volonté de créer un espace en mouvement par l'utilisation de formes de croissance et de formes courbes. L'alliance entre forme et lumière permet de générer le mouvement. Ainsi, le mur concave compresse l'espace intérieur depuis le centre où est placé l'autel et dirige celui-ci à travers les ouvertures vers l'extérieur. La lumière accentue la présence centrale de l'autel et génère un champ qui se dissipe vers l'extérieur. Finalement, cette recherche de continuité et de mouvement permet de générer une intériorité, qui constitue l'essentiel d'une église.

Benjamin CHAVARDES

architecte HMONP

enseignant à l'École nationale supérieure d'architecture de Lyon membre du laboratoire ART-Dev UMR 5281 